\title{
CARACTERÍSTICAS FÍSICAS E QUÍMICAS DOS FRUTOS DO CAMBUCIZEIRO (Campomanesia phaea) ${ }^{1}$
}

\author{
MARIA ISABEL VALLILO², MARIA LIMA GARBELOTTI ${ }^{3}$, ELISABETH DE OLIVEIRA4, \\ LEDA CONCEIÇÃO ANTONIA LAMARDO
}

\begin{abstract}
RESUMO -Frutos de Campomanesia phaea (Myrtaceae) são muito procurados pela população rural para preparo de sucos, sorvetes e bebidas alcoólicas. Para avaliar as características físicas, o potencial nutricional e o seu aproveitamento na indústria de alimentos, frutos nos seus diversos estádios de amadurecimento foram coletados em abril de 2003 no Parque Estadual da Serra do Mar, Núcleo Caraguatatuba-SP. Os dados do diâmetro longitudinal (DL) e transversal (DT) e a relação entre eles mostram que os frutos têm forma levemente achatada (DL/DT <1); a polpa mostrou-se suculenta, com sabor acre e odor cítrico, com alto teor de umidade $(88,80 \%)$ e $\mathrm{pH}$ igual a 2,91. Foram detectados elevados teores de fibras alimentares $(4,00 \%)$, quando comparado a outras espécies popularmente conhecidas, da mesma família botânica. Foram obtidos valores baixos de proteína

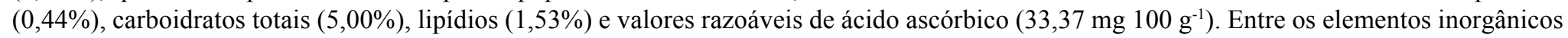
determinados (13), destacaram-se: sódio (171,50 $\left.\mathrm{mg} \mathrm{kg}^{-1}\right)$, potássio $\left(622,65 \mathrm{mg} \mathrm{kg}^{-1}\right)$, fósforo $\left(123,69 \mathrm{mg} \mathrm{kg}^{-1}\right)$, magnésio $\left(42,08 \mathrm{mg} \mathrm{kg}^{-1}\right)$ e cálcio $(61,26$ $\left.\mathrm{mg} \mathrm{kg}^{-1}\right)$. Embora a composição química dos frutos mostrou ser semelhante à de outras espécies da família Myrtacease, o consumo in natura deste fruto é prejudicado pelo baixo teor de carboidratos e elevada acidez.
\end{abstract}

Palavras-chave: Myrtaceae; cambuci; frutos; características físicas; composição química; polpa.

\section{PHYSICAL AND CHEMICAL CHARACTERISTICS OF CAMBUCIZEIRO'S FRUITS (Campomanesia phaea)}

ABSTRACT - Fruits of Campomanesia phaea (Myrtaceae) are used by rural population to make juice, ice cream and in alcoholic drinks to accentuate the flavor. To evaluate the physical characteristics, the nutritional potential and the use in food industry, fruits samples in several ripe steps were collected in April 2003 in Serra do Mar State Park, Núcleo Caraguatatuba, SP. The data of longitudinal (DL) and traverse (DT) diameter and the relationship among them showed that the fruits present oval and flattened shape (DL/DT $<1$ ); the pulp was succulent, with pungent flavor and citric scent, with high level of humidity $(88,80 \%)$ and $\mathrm{pH} 2,91$, high levels of alimentary fibers were detected $(4,00 \%)$, when compared to other species of the same botanical family. Low contents of protein $(0,44 \%)$, total carbohydrates $(5,00 \%)$, lipids $(1,53 \%)$ and reasonable level of ascorbic acid (33,37 mg $\left.100 \mathrm{~g}^{-1}\right)$ were presented. Among the inorganic elements (13) the nutrients sodium (171, $\left.50 \mathrm{mg} \mathrm{kg}^{-1}\right)$, potassium (622, $\left.65 \mathrm{mg} \mathrm{kg}^{-1}\right)$, phosphorous $\left(123,69 \mathrm{mg} \mathrm{kg}^{-1}\right)$, magnesium $\left(42,08 \mathrm{mg} \mathrm{kg}^{-1}\right)$ and calcium $\left(61,26 \mathrm{mg} \mathrm{kg}^{-1}\right)$ can be pointed out. Although the chemical composition is similar to others species from the Myrtaceae family the in natura intake of this fruit is difficult due to the low level of carbohydrates and high acid content.

Index terms: Myrtaceae; cambuci, fruits, physical characteristics, chemical composition; pulp.

\section{INTRODUÇÃO}

A família Myrtaceae Jussieu compreende 3.500 espécies, subordinadas a 100 gêneros. Seus representantes apresentam porte arbustivo ou arbóreo (Barroso, 1984).

Segundo Gemtchüjnicov (1976) e Legrand \& Klein (1977), a família é dividida em duas subfamílias, a saber:

1 - Leptospermoideae, cujos representantes apresentam frutos secos do tipo cápsula, aquênio e pixídio. Sua maior dispersão se dá na Austrália e Polinésia, e baciformes.

2 - Myrtoideae, que fornecem frutos carnosos, geralmente

O gênero Campomanesia, a que pertence a espécie em estudo, está incluído na subfamília Myrtoideae (Cronquist, 1968, 1984; Landrun, 1986).

As espécies desse gênero possuem importância econômica diversificada. Seus frutos comestíveis são consumidos por várias espécies de pássaros e mamíferos, sendo também usados na produção de doces caseiros, sorvetes, aguardente, licores e refrescos.

A espécie Campomanesia phaea (O. Berg.) Landrum é popularmente conhecida como "cambuci" ou "cambucizeiro", tendo como sinonímia botânica a designação de Paivaea langsdorffii Berg. Apresenta altura de 3-5 m e tronco com diâmetro de 20 a $30 \mathrm{~cm}$ com aspecto de escamas. Ocorre nos Estados de São Paulo (principalmente na Serra do Mar) e em Minas Gerais (Lorenzi,1992). A espécie apresenta floração branca e vistosa nos meses de agosto a novembro, e frutificação abundante, com frutos arredondados, durante os meses de janeiro e fevereiro. Quando maduros, são amarelados a alaranjados, dando à árvore aspecto de grande beleza que, somado ao seu pequeno porte, a indicam para arborização de ruas estreitas e sob redes elétricas, bem como para compor praças e jardins. É recomendada para reflorestamentos heterogêneos destinados à recomposição de áreas degradadas de preservação permanente (Lorenzi, 1992).

Os frutos medem, geralmente, de 5 a $6 \mathrm{~cm}$ de diâmetro na região mediana, por 3 a 4,5 cm de espessura; são carnosos, suculentos e comestíveis, utilizados principalmente na forma de sucos, sendo também muito apreciados pela avifauna silvestre (Lorenzi, 1992; Centro de Pesquisas Eco-Naturais - Cepen, 2003 e Jorge, 1992).

Considerações feitas por Holanda, apud Adati (2001), indicam que os frutos são considerados exóticos, possuindo interessantes propriedades aromáticas que os favorecem como agentes flavorizantes em alimentos e bebidas.

Quanto à composição química, tem-se conhecimento de estudos fitoquímicos e farmacológicos realizados por Adati (2001) nas folhas dessa espécie, detectando-se grande quantidade de óleo essencial, rico em linalol (11,11\%), óxido de cariofileno (11,77\%), betacariofileno $(6,33 \%)$, betasselineno $(6,33 \%)$ e alfacadinol $(1,94 \%)$, constituintes que apresentam grande valor comercial para as indústrias de cosméticos e farmacêuticas.

Estudos morfo-histológicos dos frutos, realizados por Jorge (1992), mostraram a existência de células pétreas no mesocarpo cujo formato e dimensões apresentam interesse para a diagnose específica.

\footnotetext{
${ }^{1}$ (Trabalho 041/2005). Recebido: 10/03/2005. Aceito para publicação: 01/08/2005. Parte do trabalho apresentado no V Encontro do Instituto Adolfo Lutz, realizado em São Paulo-SP, no período de 13 a 16 de outubro de 2003.

${ }^{2}$ Pesquisadora Científica do Instituto Florestal, Caixa Postal 1322, 01059-970, São Paulo-SP, Brasil, tel: (011) 6231-8555 - ramal: 2100. e-mail: maria-vallilo@ig.com.br. ${ }_{3}^{3}$ Pesquisadora Científica do Instituto Adolfo Lutz, Caixa Postal 355, 01246-902, São Paulo-SP, Brasil, tel: (011) 3068-2922. e-mail: 1lamardo@ial.sp.gov.br.

${ }^{4}$ Professor (a) Titular do Instituto de Química da Universidade de São Paulo, Caixa Postal 26077, 05513-970, São Paulo-SP, Brasil, tel: (011) $3091-3837$ ramal: 240. e-mail: edolivei@iq.usp.br.
} 
Os estômatos e os tricomas da polpa são diferenciados daqueles observados nas folhas.

O pouco conhecimento sobre a composição química dos frutos desta espécie motivou seu estudo, pois, além de apresentar importante potencial econômico, poderá subsidiar o cultivo e a seleção adequada visando ao seu aproveitamento na indústria de alimentos.

Portanto, o objetivo deste trabalho foi avaliar algumas características físicas, a composição química e o potencial nutricional dos frutos de C. phaea (O. Berg.) Landrum e comparar os resultados obtidos com os dos frutos de espécies popularmente conhecidas e utilizadas como alimento, pertencentes à mesma família botânica.

\section{MATERIAL E MÉTODOS}

As amostras de fruto de C. phaea foram coletadas no Núcleo Caraguatatuba, que integra a rede de Unidades de Conservação administrada pelo Instituto Florestal da Secretaria do Meio Ambiente de São Paulo. Abrange uma área de 13.769,60 ha, fazendo parte do Parque Estadual da Serra do Mar, e tendo como limites geográficos os municípios de Caraguatatuba, São Sebastião, Paraibuna, Salesópolis e Natividade da Serra. Situa-se entre as coordenadas geográficas $23^{\circ} 31^{\prime}$ a $23^{\circ} 22^{\prime}$ de Lat. S e $45^{\circ} 18^{\prime}$ a $45^{\circ} 44^{\prime}$ de Long.W. Apresenta relevo de escarpas festonadas, com altitudes que variam entre 50 e aproximadamente 1.000 metros. O solo é do tipo argilo-arenoso. A vegetação predominante é típica de floresta ombrófila densa, e a fauna é representada por muitas espécies endêmicas da Mata Atlântica e ameaçadas de extinção, como o bugio (Alouatta fusca), a onça-parda (Felis concolor), o cachorro-do-mato (Speothos venaticus), o jacu (Penélope ochrogaster), o gavião-pombo (Leucopternis sp), entre outros (Núcleo, 19...).

Frutos inteiros de C. phaea (40 unidades), em seus vários estádios de amadurecimento e provenientes de três plantas produtivas, localizadas no interior da mata, recebendo parcialmente a luz solar, foram coletados em abril de 2003, acondicionados em sacos de polietileno e guardados sob congelamento à temperatura de $5^{\circ} \mathrm{C} \pm 2^{\circ} \mathrm{C}$, por aproximadamente uma semana, para posterior análise nos laboratórios do Instituto Florestal, do Instituto Adolfo Lutz e do Instituto de Química da Universidade de São Paulo.

Para as determinações das características físicas, separaramse, aleatoriamente, 16 frutos, que foram avaliados individualmente através do seu peso e dos diâmetros longitudinal (DL) e transversal (DT), sendo este último avaliado na parte mais larga do fruto, através de paquímetro digital. O rendimento em polpa, casca e sementes foi obtido pela relação percentual entre o peso do fruto inteiro e de suas respectivas estruturas.

Para as análises químicas, as amostras (24 frutos) foram trituradas e homogeneizadas integralmente em multiprocessador doméstico, constituindo uma amostra composta dos vários frutos. As análises, em triplicata, foram feitas segundo as "Normas Analíticas do Instituto Adolfo Lutz" (Instituto Adolfo Lutz, 1985), sendo as fibras alimentares totais dosadas pelo método enzimático-gravimétrico da "Association of Official Analytical Chemists - AOAC", modificado por Lee et al. (1992), e o ácido ascórbico (vitamina C), pelo método descrito por Contreras-Guzmán et al. (1984).

O valor calórico foi calculado utilizando-se dos seguintes fatores clássicos de conversão de Atwater: $9 \mathrm{kcal}$ por $\mathrm{g}$ de lipídios, 4 kcal por g de proteínas e $4 \mathrm{kcal}$ por $\mathrm{g}$ de carboidratos (De Angelis, 1977).

A determinação dos elementos inorgânicos $(\mathrm{Na}, \mathrm{K}, \mathrm{P}, \mathrm{Ca}, \mathrm{Mg}$, $\mathrm{V}, \mathrm{Mn}, \mathrm{Fe}, \mathrm{Ni}, \mathrm{Cu}, \mathrm{Zn}, \mathrm{Pb}$ e $\mathrm{Al}$ ) foi realizada no laboratório de espectrometria do Instituto de Química da Universidade de São Paulo, após digestão ácida da amostra $\left(\mathrm{HNO}_{3}\right.$ a $\left.30 \%\right)$, em sistema focalizado Spex 350, assistida por radiação de microondas, com nove repetições analíticas e determinação por ICP-OES, conforme descrito por Vallilo et al. (2003).

\section{RESULTADOS E DISCUSSÃO}

Os frutos de C. phaea apresentam cor verde a verde-amarelada, exalando aroma cítrico, levemente adocicado, persistente e bastante agradável ao olfato. Segundo Cipollini \& Stile (1993), as características físicas dos frutos, como cor, tamanho e número de sementes, bem como a quantidade de polpa e o conteúdo de água, podem influenciar no seu consumo, tanto como alimento da avifauna quanto pelo homem. Herrera (1982) salienta, contudo, que os elementos nutritivos, que são importantes para as aves, incluem carboidratos, minerais, lipídios e proteínas.

No entanto, para a fabricação industrial de sucos, sorvetes e principalmente de doces em calda ou glaciados, onde a aparência do produto final é primordial, algumas características físicas devem ser determinadas e avaliadas. Nesse sentido, são apresentados, na Tabela 1, alguns parâmetros físicos nos quais se pode observar uma grande variação em relação às massas frescas dos frutos $(38,7 \mathrm{~g}$ a 75,3 g) e de suas principais estruturas: casca, polpa e sementes, apresentando, em média, $18,65 \%, 80,56 \%$, e $0,79 \%$, respectivamente. Foram avaliados 16 frutos.

TABELA 1 - Características físicas de frutos de cambuci (Campomanesia phaea), colhidos no Parque Estadual da Serra do Mar, Núcleo Caraguatatuba-SP, em abril de 2003.

\begin{tabular}{cccc}
\hline & Valor & Valor & Valor \\
Características & Médio* & Mínimo & Máximo \\
\hline Fruto inteiro (g) & 55,81 & 38,70 & 76,28 \\
Polpa (\%) & 80,56 & 46,39 & 92,53 \\
Casca (\%) & 18,65 & 13,90 & 22,85 \\
Sementes (\%) & 0,79 & 0,13 & 1,12 \\
$\quad$ Diâmetro & & & \\
Longitudinal (DL) & 42,60 & 38,11 & 48,00 \\
$\quad$ em mm & & & \\
Diâmetro & & & \\
Transversal (DT) & 51,27 & 46,01 & 61,51 \\
em mm & & & \\
DL/DT & 0,84 & - & -
\end{tabular}

* Número total de frutos avaliados: 16 .

Segundo Andrade et al. (1993), essa variabilidade na massa fresca e nos rendimentos de suas partes interfere na eficiência dos processos industriais para fabricação de doces, exigindo uma adequada classificação ou separação prévia dos frutos por tamanho ou peso.

Os dados do diâmetro longitudinal (DL) e transversal (DT) e a relação entre eles (DL/DT) mostram que os frutos de $C$. phaea têm forma levemente achatada (DL/DT $<1)$, característica dessa espécie botânica. Para a fabricação de doces em calda ou glaciados, normalmente, se dá preferência a frutos com uniformidade de formato (levemente arredondado) (DL/DT = 1) (Andrade et al., 1993).

A polpa caracteriza-se por apresentar alto teor de umidade $(88,80 \%)$, o que indica ser uma das características comuns de frutos da família Myrtaceae, enquadrando-se na classe dos frutos carnosos e suculentos e confirmando a descrição feita por Gemtchüjnicov (1976) e Legrand \& Klein (1977), quanto às características de frutos da subfamília Myrtoideae (Tabela 2).

Comparando-se a composição química de C. phaea com as das demais espécies listadas na Tabela 2 , verificou-se a presença de alto teor de lipídios (1,53\%) que, somado ao teor de carboidratos $(5,00 \%)$, indicam valor energético de $35,53 \mathrm{kcal} 100 \mathrm{~g}^{-1}$, sendo que o conteúdo de proteína $(0,44 \%)$ pouco contribui nesse sentido.

Os frutos são ácidos ( $\mathrm{pH}=2,91)$, favorecendo os processos de industrialização na forma de doces, porém inibindo o seu consumo in natura. Segundo Andrade et al. (1993), na indústria de sucos, o alto teor de acidez provoca elevada diluição do produto e, por conseguinte, 
TABELA 2 - Composição centesimal, valor calórico total (V.C.T.) e teor de ácido ascórbico da polpa de cambuci (Campomanesia phaea), pêra do campo (Eugenia Klotzschiana), jabuticaba (Myrciaria trunciflora Berg.), kiwi (Actinidia chinensis), pitanga (Eugenia uniflora L.) goiaba (Psidium guajava L.), uvaia (Eugenia pyriformis Camb.), jambo (Syzigium jambos L.).

\begin{tabular}{|c|c|c|c|c|c|c|c|c|c|c|}
\hline $\begin{array}{c}\text { Composição } \\
(\%)\end{array}$ & $\mathrm{Camb}$ & uci** & $\begin{array}{l}\text { Pêra do } \\
\text { Campo }^{3}\end{array}$ & $\begin{array}{c}\text { Goiaba } \\
\text { branca }\end{array}$ & $\begin{array}{c}\text { Goiaba } \\
\text { vermelha }^{1,2}\end{array}$ & Jabuticaba $^{1,2}$ & Kiwi $^{1,2}$ & Pitanga $^{1,2}$ & Uvaia $^{1,2}$ & Jambo $^{1,2}$ \\
\hline Umidade & 88,8 & 0,2 & 88,30 & 86,07 & 85,81 & 87,85 & 83,06 & 90,47 & 85,53 & -- \\
\hline Resíduo mineral fixa & 0,23 & 0,01 & 0,04 & 0,44 & 3,27 & 2,08 & 0,52 & 0,28 & 0,44 & -- \\
\hline Lipídios & 1,53 & 0,09 & 0,80 & 0,54 & 0,64 & 0,89 & 1,37 & 0,23 & 2,04 & 0,20 \\
\hline Proteínas & 0,44 & 0,04 & 0,60 & 0,68 & 0,76 & 0,22 & 1,16 & 0,76 & 1,56 & 0,80 \\
\hline Fibras & 4,00 & 0,08 & 4,00 & 5,63 & 4,95 & 2,06 & 2,30 & 2,10 & 2,04 & -- \\
\hline Carboidratos Totais* & 5,00 & 0,03 & 6,26 & 12,27 & 9,52 & 8,96 & 13,89 & 8,26 & 10,43 & 12,8 \\
\hline $\begin{array}{c}\text { Ácido ascórbico } \\
\left(\mathrm{mg} 100 \mathrm{~g}^{-1}\right) \\
\text { pH em solução aquosa, }\end{array}$ & 33 & 1 & 32,80 & -- & $40,0-80,1^{4}$ & 12,8 & $98^{5}$ & 14,0 & -- & 20,0 \\
\hline $\begin{array}{l}\left.\left(1: 10 \mathrm{~mL}^{-1}\right) ; \mathrm{T}=20 \mathrm{C}\right) \\
\text { Acidez em ácido cítrico }\end{array}$ & $\begin{array}{r}2,91 \\
3,0\end{array}$ & $\begin{array}{l}0,01 \\
0,1\end{array}$ & 2,54 & -- & -- & -- & -- & -- & -- & -- \\
\hline V.C.T $\left(\right.$ kcal $\left.100 \mathrm{~g}^{-1}\right)$ & 35,5 & 0,9 & 35,0 & 34,0 & 27,0 & 36,0 & 63,0 & 30,0 & 58,0 & 50,0 \\
\hline
\end{tabular}

maior rendimento final do produto.

O teor de ácido ascórbico encontrado supera os das espécies da família Myrtaceae, apresentadas na Tabela 2, com exceção da goiaba vermelha e kiwi. No entanto, também contribui para acentuar o sabor típico do fruto. Considera-se razoável o valor encontrado $(33,37 \mathrm{mg}$ $100 \mathrm{~g}^{-1}$ ), visto que a legislação brasileira (Brasil, 1998a) recomenda a ingestão diária de $60 \mathrm{mg}$ por dia, para adultos. Porém, verifica-se que cada fruto de $C$. phaea, pesando em média $55,81 \mathrm{~g}$, fornece $31,03 \%$ de ácido ascórbico. Seu teor equipara-se ao da polpa da Eugenia klotzschiana Berg (pêra do campo), tornando essa fruta uma fonte alternativa natural desse ácido para a avifauna e população da região. No entanto, o valor encontrado varia conforme o ponto de maturação, os fatores ambientais e as manipulações e estocagens inadequadas dos frutos.

Em relação ao teor de fibra alimentar encontrado (4,00 \%), assemelha-se aos da pêra do campo e das goiabas branca e vermelha. Considerando-se a ingestão diária do fruto, com peso médio de $55,81 \mathrm{~g}$, verificou-se que ele contribui, em média, com $11 \%$ de fibras na dieta alimentar, quando se toma como base o valor recomendado pela Sociedade Brasileira de Nutrição (20 g por dia).

TABELA 3 - Teores dos elementos inorgânicos nos frutos inteiros de Campomanesia phaea (cambuci), expressos mg. $\mathrm{kg}^{-1} \mathrm{e}$ respectivo desvio-padrão; contribuição por fruto, expressos em percentagem.

\begin{tabular}{|c|c|c|c|c|c|c|}
\hline & $\begin{array}{c}\text { Cam } \\
\text { Mé } \\
(\end{array}$ & $\begin{array}{l}\text { buci } \\
\text { dia } \\
*) \\
\end{array}$ & $\begin{array}{c}\text { (\%)/ Fruto } \\
(55,81 \mathrm{~g}) \\
\text { (Cambuci) }\end{array}$ & Jabuticaba & Kiwi $^{1}$ & Pitanga \\
\hline $\mathrm{Na}$ & 172 & 4 & 0,31 & n.d. & 50 & 30 \\
\hline $\mathrm{K}$ & 623 & 6 & 1,12 & 3030 & 3320 & 1030 \\
\hline $\mathrm{P}$ & 124 & 1 & 0,22 & 140 & 400 & 110 \\
\hline $\mathrm{Ca}$ & 61,3 & 0,5 & 0,11 & 130 & 260 & 90 \\
\hline $\mathrm{Mg}$ & 42,1 & 0,1 & 0,08 & n.d. & 300 & 120 \\
\hline V & 1,9 & 0,3 & 0,003 & n.d. & n.d. & n.d. \\
\hline $\mathrm{Mn}$ & 0,27 & 0,05 & 0,0005 & n.d. & n.d. & n.d. \\
\hline $\mathrm{Fe}$ & 3,60 & 0,04 & 0,006 & 19 & 9,1 & 2,0 \\
\hline $\mathrm{Ni}$ & 0,17 & 0,01 & 0,0003 & n.d. & n.d. & n.d. \\
\hline $\mathrm{Cu}$ & 1,46 & 0,02 & 0,003 & n.d. & 0,5 & 2,0 \\
\hline $\mathrm{Zn}$ & 3,46 & 0,08 & 0,006 & n.d. & 1,0 & n.d. \\
\hline $\mathrm{Pb}$ & 1,63 & 0,03 & 0,003 & n.d. & n.d. & n.d. \\
\hline $\mathrm{Al}$ & 9,6 & 0,2 & 0,02 & n.d. & n.d. & n.d. \\
\hline
\end{tabular}

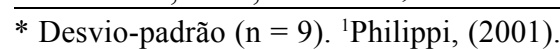

n.d. não determinado.
Em relação aos minerais (Tabela 3), o potássio predominou em quantidade sobre os demais, seguido de sódio, fósforo e cálcio. Sabese que tanto o sódio quanto o potássio são largamente distribuídos na natureza, o mesmo não ocorrendo com o fósforo. Esse, muitas vezes, está no solo numa estrutura química pouco solúvel e, portanto, pouco assimilável pela planta. Porém, os teores encontrados podem estar associados à localização dos pontos de coleta (Núcleo Caraguatatuba) no Parque Estadual da Serra do Mar, cuja proximidade com o oceano pode estar sofrendo influência direta e freqüente da neblina, ventos e chuvas, carreando, dessa forma, os elementos químicos para esse ecossistema, fato observado para o teor de $\mathrm{Na}$, que se encontra de 3 a 5 vezes maior que, para os teores, para os frutos relatados na literatura (Philippi, S. T., 2001). Já os teores encontrados para os micronutrientes potencialmente tóxicos estão compatíveis com os valores permitidos pela legislação vigente (Brasil, 1998b), que estabelece limites máximos de tolerância para contaminantes inorgânicos em alimentos, principalmente para cobre $\left(10 \mathrm{mg} \mathrm{kg}^{-1}\right)$ e chumbo $\left(0,3 \mathrm{mg} \mathrm{kg}^{-1}\right)$, este referenciado somente para sucos de frutas cítricas. Pela contribuição de cada fruto (Tabela 3), pode-se inferir que os frutos de C. phaea, coletados nessa região, não apresentam toxicidade, podendo fazer parte da alimentação de vertebrados e ser aproveitado na indústria de alimentos. Os teores de nutrientes, encontrados no presente trabalho, poderão ser utilizados na elaboração de dietas de indivíduos saudáveis (Philippi, S. T., 2001).

\section{CONCLUSÕES}

O fruto de $C$. phaea (cambuci) apresenta limitações ao consumo in natura devido ao baixo teor de carboidratos e elevada acidez. Apesar de não mostrar uniformidade no seu formato, apresenta potencial para a industrialização devido aos seus atributos de qualidade, como alto rendimento em polpa, elevada acidez, razoáveis concentrações de ácido ascórbico, minerais e fibras alimentares. De maneira geral, a composição química mostrou ser semelhante à das outras espécies da família Myrtaceae, popularmente conhecidas e utilizadas como alimento pela população.

\section{AGRADECIMENTOS}

Aos pesquisadores Ivan Suarez da Mota, do Núcleo Caraguatatuba (Parque Estadual da Serra do Mar, Instituto Florestal de São Paulo), pelo envio do material botânico; à Sra. Yara Cristina Marcondes, do Instituto Florestal de São Paulo, pela correção do texto, e ao Sr. Leonídio Guilherme, da Seção de Doces do Instituto Adolfo 
Lutz, pelo auxílio nas análises bromatológicas.

\section{REFERÊNCIAS}

ADATI, R. T. Estudo biofarmagnóstico de Campomanesia phaea (O. Berg. ) Landrum. Myrtaceae. 2001. 128f. Dissertação (Mestrado em Farmacognosia)- Faculdade de Ciências Farmacêuticas, Universidade de São Paulo, São Paulo, 2001.

ANDRADE, J. de S.; ARAGÃO, C. G.; FERREIRA, S. A. do N. Caracterização física e química dos frutos de Araçá-Pêra (Psidium acutangulum) D. C.). Acta Amazônica, Manaus, v. 23, n. 2-3, p. 213 217, 1993.

BARROSO, G.M.; PEIXOTO, A. L. Sistemática de angiospermas do Brasil. Viçosa: Ed. Universidade Federal de Viçosa, 1984. v.2, 377p.

BRASIL. Portaria n ${ }^{\circ} 33$ de 13 de janeiro de 1998 do Ministério da Saúde. Princípios gerais para o estabelecimento de níveis máximos de ingestão diária em alimentos. Diário Oficial da União, Brasília, DF, 16 jan. 1998a Seção I-E, p. 5.

BRASIL. Portaria ${ }^{\circ} 685$ de 27 de agosto de 1995, da Agência Nacional de Vigilância Sanitária (ANVISA). Princípios gerais para o estabelecimento de níveis máximos de contaminantes químicos em alimento. Diário Oficial da União, Brasília, DF, 24 set.1998b. Seção $1, \mathrm{n}^{\circ} 183 \mathrm{E}$, p. 03.

BRASIL. Instrução Normativa $n^{\circ} 1$ de 7 de janeiro de 2000 do Ministério de Agricultura e Abastecimento. Aprova regulamento técnico geral para a fixação dos padrões de identidade e qualidade para polpa de frutas. Diário Oficial da União, Brasília, DF., 10 jan. 2000. Seção 1, p. 54-58.

CENTRO DE PESQUISAS ECO-NATURAIS - CEPEN. Cambuci, Campomanesia phaea. Disponível em: <http:// www.cepen.com.br/arv_nat_Myrtaceae.htm>. Acesso em: 07 out. 2003.

CIPOLLINI, M. L.; STILES, E. W. Fruit rot, antifungal defense, and palatability of fleshy fruits for frugivorous birds. Ecology, Washington, D.C., v. 74, n. 3, p. 751-762, 1993.

CONTRERAS-GUZMÁN, E. S.; STRONG III, F. C.; GUERNELLI, O. Determinação de ácido ascórbico (vitamina $C$ ) por redução de íons cúpricos. Química Nova, São Paulo, v. 7, n. 2, p. 60-64, 1984.

CRONQUIST, A. The evolution and classification of flowering plants. Boston: Houghton Mifflin, 1968, 396p.

CRONQUIST, A. A commentary of the definition of order Myrtales. Ann. Missouri Bot. Gard., Missouri, v. 71, p. 780-782, 1984.
DE ANGELIS, R. C. Fisiologia da nutrição: fundamentos para nutrição e desnutrição. São Paulo: EDART/EDUSP, 1977. v. 1.

FRANCO, G. Tabela de composição química de alimentos. 9. ed. Rio de Janeiro: Atheneu, 1992.305p.

GEMTCHÜJNICOV, I. D. Manual de taxonomia vegetal: plantas de interesse econômico, agrícola, ornamentais e medicinais. São Paulo: Ceres, 1976.368p.

HERRERA, C. M. Seasonal variation in the quality of fruits and diffuse coevolution between plants and avian dispersers. Ecology, Washington, D.C, v. 63, n. 3, p. 773-785, 1982.

INSTITUTO ADOLFO LUTZ. Normas analíticas do Instituto Adolfo Lutz. 3. ed. São Paulo: IMESP, 1985. v. 1. 533p.

JORGE, L. I. F. Caracterização farmacobotânica e microscópica alimentar de seis espécies brasileiras de Myrtaceae JUSSIEU. 1992. 140 f. Dissertação (Mestrado em Farmacognosia) - Faculdade de Ciências Farmacêuticas, Universidade de São Paulo, São Paulo, 1992.

LAJOLO, M. F. Tabela brasileira de composição de alimentos. Disponível em <www.fcf.usp.tabela >. Acesso em: 03 dez. 2001.

LANDRUM, L. R. Campomanesia. In: FLORA neotrópica. New York: The New York Botanical Garden, 1986. v. 45, p. 7-72.

LEE, S.C.; PROSKY, L.; DEVRIES, J.W. Determination of total, soluble and insoluble dietary fiber in foods. Enzimatic-gravimetric method, Mês-TRI Buffer: colaborative study. Journal of the Association of Official Analytical Chemists International, Gaithersburg, v. 75, p. 395-416, 1992.

LEGRAND, C. D.; KLEIN, R. M. Mirtáceas: Campomanesia, In: REIZ, P.R. Flora ilustrada catarinense. Itajaí: Herbário Barbosa Rodrigues, 1977.p. 573-623.

LORENZI, H. Árvores brasileiras: manual de identificação e cultivo de plantas arbóreas nativas do Brasil. Nova Odessa: Plantarum, 1992. $252 \mathrm{p}$.

NÚCLEO Caraguatatuba: Parque Estadual da Serra do Mar. São Paulo: Instituto Florestal, [19...]. Não paginado. (Série Áreas Naturais, 10).

PHILIPPI, S.T. Tabela de Composição de Alimentos: suporte para decisão nutricional. Brasília: ANVISA, FINATEC/NUT-UnB, 2001.133p.

VALLILO, M. I.; BAITELLO, J. B.; LAMARDO, L.; LOBANCO, C. M. Composição química do fruto de Eugenia klotzschiana Berg. (MYRTACEAE). Revista do Instituto Florestal, São Paulo, v. 15, n. 1, p. 33-44, jun. 2003. 